\title{
Device Control using Intelligent Switch
}

\author{
M.V. Sreenivas Rao ${ }^{1}$, Basavanna $\mathbf{M}^{2}$ \\ Associate Professor, Dept of Instrumentation Technology, GSSSIETW, Mysuru ${ }^{1}$ \\ Assistant Professor, Dept of Instrumentation Technology, GSSSIETW, Mysuru ${ }^{2}$
}

\begin{abstract}
Intelligent switch is capacitive touch sensor which senses the touch. This type of switches takes body capacitance as a input in order to turn ON or OFF the devices which is connected to it and also the regulation of it. The devices controlled are bulb, fan, motor and other electronic appliances. This touch switches are needed in order to overcome the disadvantages of mechanical switches like wear and tare, limited operations, sparks etc. The capacitive touch sense pad which consists of one or more sense buttons. When the person touches the touch pad it takes the body capacitance and that analog signal is converted to digital signal in order to make compatible for the MSP430G2553 microcontroller to operate. This microcontroller contains pin oscillator and PWM which is used to turn ON or OFF and regulation of the particular devices respectively. Finally, the capacitive touch sensor are used to replace the mechanical switches thus providing high reliability, shock proof, trendy looks etc. The regulation of the devices which is connected to these switches can also be done using the same switches. Here regulation means light intensity control in the in bulb, speed control in the case of motor etc. Thus it reduces the power consumption and improves the quality of life.
\end{abstract}

Keywords: Capacitive Touch Sensor, Pulse Width Modulation (PWM), Light Emitting Diode (LED), DC Gear Motor.

\section{INTRODUCTION}

Automation is the delegation of human control functions to technical equipment. Control of any system is achieved by making the system smart. Capacitive Touch Sensing, is based on the concept of measuring the altered capacitance of a sensor or touch pad in the presence of a pressure source. The Capacitive touch sensing is more advantageous than mechanical switches. Touch switches avoids wear and tare, sparks etc. It gives a unlimited operation and consumes less power if it is implemented by using MSP430 microcontroller. The regulation is done by using PWM which is inbuilt in MSP430 microcontroller. Conventional mechanical buttons or potentiometers with moving parts have a certain lifetime. Sooner or later they are worn out and do not work reliably anymore. Due to the lack of moving parts, capacitive touch is much more durable. One positive aspect of the moving parts in conventional push buttons or switches is the tactile feedback to the user. By touching a surface, the user does not "feel" if the push button was triggered. This can be compensated by using optical, acoustical or, a bit more complex, vibration feedback.

The MSP430G2533 family includes the pin oscillator and PWM built into it. Pin oscillator, is used in capacitive sensing applications to eliminate external passive components. Additionally it may be used in other sensing applications. The basic feature of pin oscillator is that it takes capacitance as the input, as the capacitance increases oscillations decreases and vice versa [1].

Pulse Width Modulation (PWM), is a technique in which the width of a pulse is modulated keeping the time period of the wave constant. One cycle has a fixed time period called 'Period' and a varying on time called 'Duty cycle'. The entire wave can have two voltages levels either logic 0 or logic 1 . The PWM wave is very useful in the digital systems, since this can be used to generate different voltage values other than the logic 0 or logic 1 values. This feature is used in many digital systems like DC motor speed control, volume control in audio devices, decoration light controls etc.[2]

\section{OBJECTIVE}

Capacitive touch is sometimes considered more art than science. Capacitive touch sensors sense the touch and perform the required task. Pin oscillator in MSP430G2553 plays a major role in providing the control, thus making it smart device control. The PWM technique is used to conserve the power, so that unnecessary wastage of power can be avoided by using the required amount of energy at the required level. The disadvantages of mechanical switches like, wear and tear, sparks, limited operations, power consumption .In order to overcome these disadvantages capacitive touch sensors can be used [4].

Capacitive touch sensors sense the touch and perform the required task. Pin oscillator and MSP430G2553 plays a major role in this project, thus creates smart home. It is used to conserve the power by using PWM technique. So that unnecessary wastage of power can be avoided by using the required amount of energy at the required level [3]. 


\section{METHODOLOGY}

Control of the devices is required as the processes will occur faster, safer, cheaper, reliably and with greater precision. Automation in home is necessary so as to control the wastage of energy. The lights and fans that will turn ON when the switch is ON so the unnecessary wastage of power can be avoided. This is a method to keep a check on the wastage of electricity as its conservation is very much needed in today's life where the resources are getting depleted.

When a person touches the touch pad the signal generated will be analog in nature, the analog signal is converted to digital signal by using analog to digital convertor. The digital signal is used to turn ON and OFF the device which is connected using pin oscillator which is inbuilt in MSP430G2553 microcontroller. And the devices are regulated by using PWM signal. The regulator is usedin.

\section{HARDWARE DETAILS}

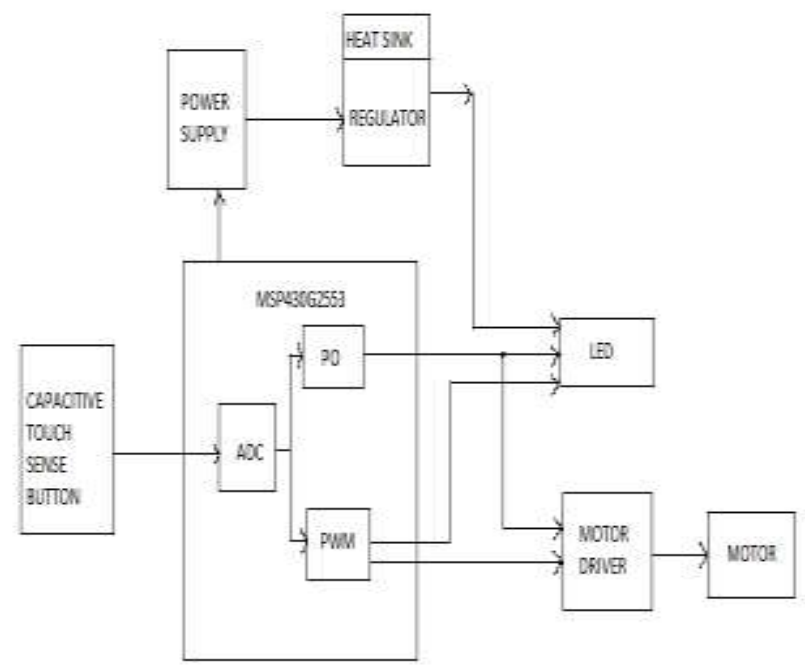

Fig 1 Block diagram of Intelligent Switch

The following are the hardware components used in this project:

A) MSP430G2553 Microcontroller

B) Touchpad

C) DC motor

D) Motor driver

A) MSP430G2553 Microcontroller

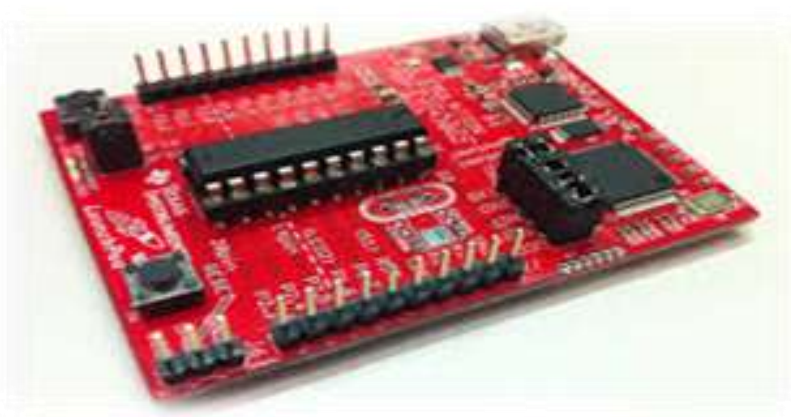

Fig 2 MSP430G2553 launch pad

The MSP430G2553 incorporates a 16bit RISC CPU,16 bit registers, 16 bit timers, peripherals and a flexible clock system that interconnect using a von-Neumann common Memory Address Bus (MAB) and Memory Data Bus (MDB). The key features of MSP430G2553 include Ultralow-power architecture which extends battery life with $0.1 \mu \mathrm{A}$ RAM retention, $0.8 \mu \mathrm{A}$ real time clock mode, $250 \mu \mathrm{A} / \mathrm{MIPS}$ active. It has high performance analog ideal for precision measurement. order to convert $12 \mathrm{v}$ to $5 \mathrm{v}$ which is needed for the LED to glow [5]. 
The MSP430G2553 microcontroller has 8kB Flash, 256kB RAM, 16GPIO and a 10 bit ADC. It has a 16-bit RISC that enables new applications at a fraction of the code size. Compact code design reduces power consumption and cost. It is optimized for modern high level programming. It includes 27 core instructions and 7 ddressing modes.

B) Touch pad

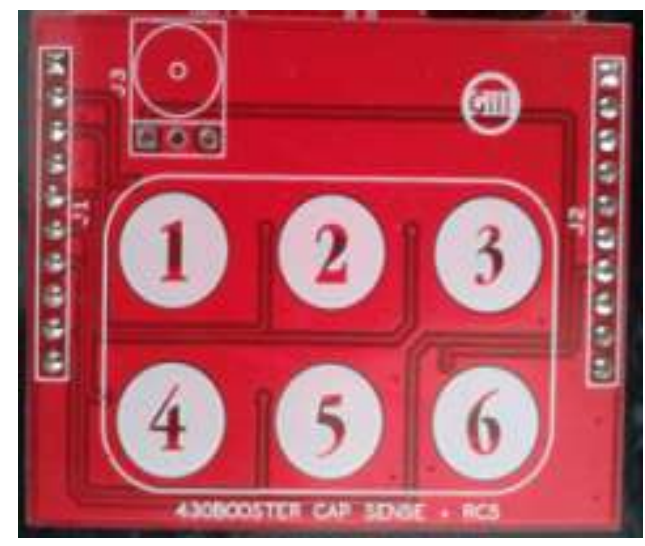

Fig3: Touch pad

The board shows 6 sensitive pad. The five different capacitive touch sensor areas are connected to the Port 2 of the device while one capacitive touch sensor area is connected to port 1.Port pin P2.1 is used as a capture pin for IR sensor and hence one pin from port 1 is used for capacitive touch sensing. On the MSP430 Value Line devices with capacitive-touch enabled I/Os, Port 2 I/Os have no analog functionality. The six LED's are connected internally to the Port 1 pins [6]. To enable the capacitive-touch feature of the I/Os, set the secondary port select PxSEL2 and clear the PXSEL bit. The selected pins start oscillating immediately, and the frequency is a direct indication of the capacitance connected to the port pin. The capacitive-touch I/Os oscillate within a frequency range of 1 to $2 \mathrm{MHz}$, which is strongly dependent on the supply voltage, the device package, and environmental influences.

Capacitive sensing may be used in many place where low to no force human touch sensing is desirable. Each pad is connected to respective pins and the internal connections are as shown in the figure.

\subsection{Geared motor}

A gear motor is a type of electrical motor. Like all electrical motors, it uses the magnetism induced by an electrical current to rotate a rotor that is connected to a shaft. The energy transferred from the rotor to the shaft is then used to power a connected device.

In a gear motor, the energy output is used to turn a series of gears in an integrated gear train. There are a number of different types of gear motors, but the most common are AC (alternating current) and DC (direct current).

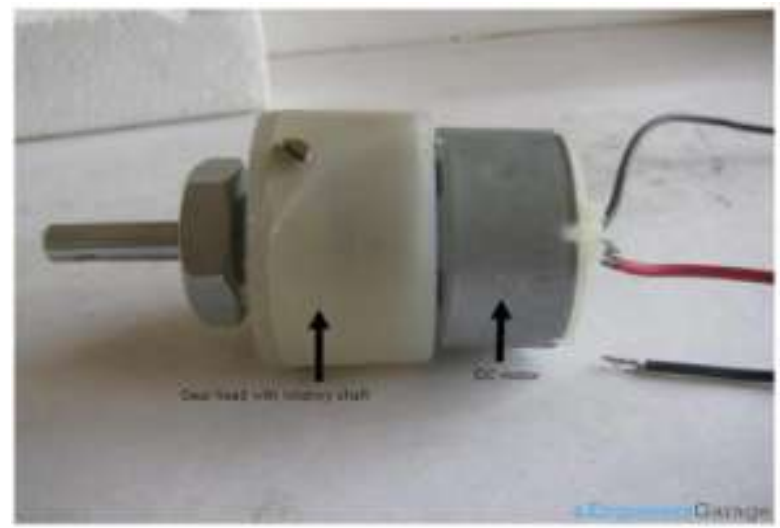

Fig 4 Geared DC motor

Geared DC motors can be defined as an extension of DC motor which already had its Insight detail demystified here. A geared DC Motor has a gear assembly attached to the motor. The speed of motor is counted in terms of rotations of the shaft per minute and is termed as RPM .The gear assembly helps in increasing the torque and reducing the speed. Using 


\section{IJARCCE}

the correct combination of gears in a gear motor, its speed can be reduced to any desirable figure. This concept where gears reduce the speed of the vehicle but increase its torque is known as gear reduction. This Insight will explore all the minor and major details that make the gear head and hence the working of geared DC motor.At the first sight, the external structure of a DC geared motor looks as a straight expansion over the simple DC ones. The lateral view of the motor shows the outer protrudes of the gear head. A nut is placed near the shaft which helps in mounting the motor to the other parts of the assembly [7].

D) Motor driver circuit

A motor driver circuit is designed to drive an electromagnetic load such as Dc gear motor, stepper motor etc.The motor driver provides the interface between the signal processing circuitry and the motor itself. It is essentially the "amplifier "for the motor. The DC motor drives are used mainly for good speed regulation, frequent starting, breaking and reversing [8].

\section{PRINCIPLE OF OPERATION}

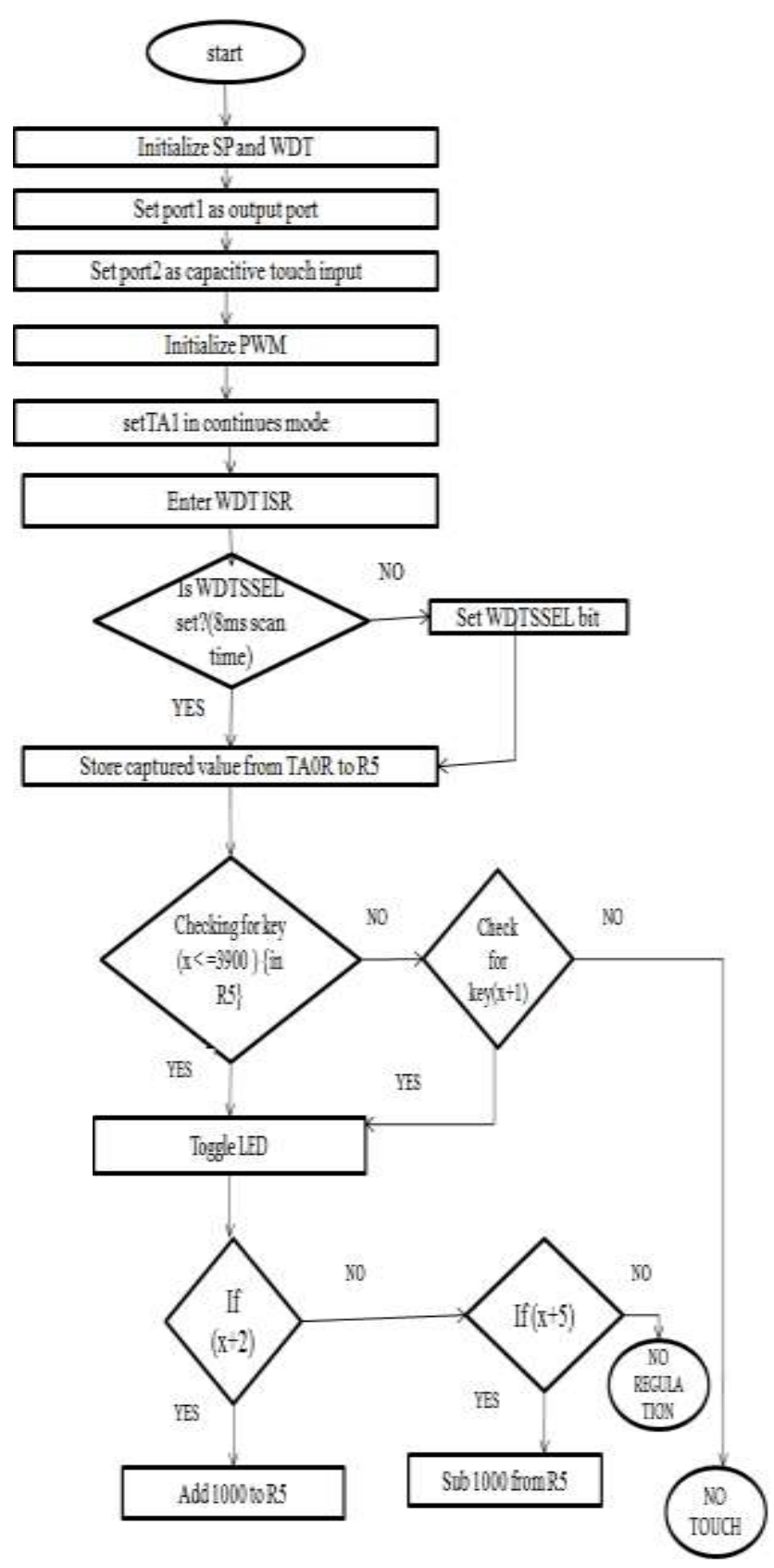

FIG 5.1 Flowchart of control unit 
The microcontroller is interfaced to the PC. In this project there are two main things that has to be consider. One is pin oscillator. The main function of pin oscillator is that it takes capacitance as the input and gives oscillations as the output. As the capacitance in the input side increases the oscillations in the output side decreases and vice versa. Second important consideration is that at the output the threshold has been set up for reference. If somebody touches the touch pad the body capacitance added with the circuit capacitance and the capacitance increases. That capacitance value increases above the threshold value which was set up earlier, the oscillations in the output decreases. If the oscillations decreases below the threshold value, the circuit decides that somebody touches the pad and it will glow that particular LED. In order to regulate the intensity ,two buttons has been set up for increasing and decreasing the intensity. This is done by pulse width modulation technique $[9,10]$

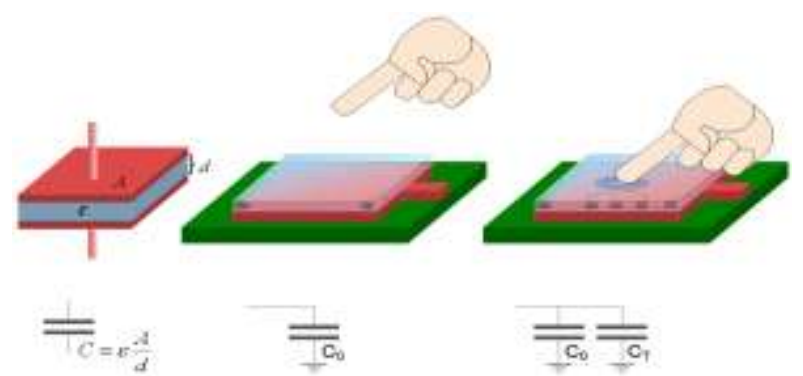

Fig 5 Basic Diagram of Capacitive touch

\section{RESULT}

Varied capacitance is taken as the input and the connected LED'S and Motors are regulated as expected, thus reduce the power consumption. The flow chart of the control unit for an intelligent switch configures the ports and enables the operation of LED as shown in Fig 5.1

Initially the input ports and output ports and PWM are initialized. Once initialization the is done it will check for 8ms time because nobody can touch and go within $8 \mathrm{~ms}$.Later it will check for the threshold 3900 .If the timer content is less than the threshold it will toggle the LED else retain its original state. For regulation purpose it will add and subtract 1000 from the timer content in order to increases and decrease the intensity and speed respectively.

\section{CONCLUSION}

Using the capacitive touch library associated with the MSP430 booster, users can manipulate the MSP430 Launch pad to distinguish between different pins. The circuit and program created in this application note were relatively simple. The main function of the program was to have the microcontroller continually check to see if the capacitance changed on any pin and perform an action based on that pin. The circuit requires a speaker and a conductive material. The simplicity of the program and circuit reflect the flexibility of the design. It can only be used to distinguish between different, isolated positions. This is unlike normal uses for capacitive touch, in which the sensors distinguish between a positions on the sensor relative to another. Capacitive touch sensing will become increasingly popular, not only for consumer products but also white goods and industrial applications, for reasons of usability, robustness and cost efficiency. The technology and available products are mature and allow the design of reliable solutions. Capacitive sensing method which offers high performance and easy, fast integration into embedded systems.

Capacitive touch sensing will become increasingly popular, not only for consumer products but also white goods and industrial applications, for reasons of usability, robustness and cost efficiency. The technology and available products are mature and allow the design of reliable solutions. Capacitive sensing method which offers high performance and easy, fast integration into embedded systems.

\section{REFERENCES}

[1] Robert E Marin, Roger K Simonson, “Capacitive Key switch Sensor and Method” US Patent \#3931610, 1976

[2] MSP430x2xx Family User's Guide (SLAU144)

[3] 3 MSP430F20xx data sheet (SLAS491)

[4] MSP430FG4618/F2013 Experimenter Board and Software http://focus.ti.com/docs/toolsw/folders/print/msp-exp430fg4618.html

[5] MSP430 USB Stick Development Tool http://focus.ti.com/docs/toolsw/folders/print/ez430- f2013.htm

[6] http://energia.nu/Serial.html

[7] http://forum.43oh.com/topic/3158-energia-library-capacitive-touch-library/

[8] http://www.embedded.com/design/mcus processors

[9] microchip Application Note 1101

[10] Guaus, E., OzaslanT., Palacios E., Arcos J.L. 2014 